\title{
PLANTEAMIENTO DE UN PROYECTO DE INCORPORACIÓN LÉXICA EN ESPAÑOL LENGUA META. CUESTIONES METODOLÓGICAS Y ESTUDIO PILOTO
}

\author{
Alicia San Mateo Valdehíta \\ Universidad Nacional de Educación a Distancia \\ asanmateo@ flog.uned.es \\ María Antonieta Andión Herrero \\ Universidad Nacional de Educación a Distancia \\ maandion@ flog.uned.es
}

\section{RESUMEN}

Este trabajo presenta el proyecto de una investigación experimental global centrada en el aprendizaje del léxico en español como lengua no materna (lengua meta), en vías de aplicación en varios puntos de actuación y diferentes contextos de aprendizaje: España, Brasil y EE. UU. Su objetivo es determinar la forma más eficaz de presentar una palabra nueva a un hablante no nativo para que sea más fácilmente incorporada a su lexicón mental; en concreto, empleamos tres actividades tipo utilizadas en la enseñanza de segundas lenguas: selección de definiciones, selección de ejemplos y escritura de oraciones. La hipótesis primera es que la tarea que le exige más grado de esfuerzo y procesamiento al aprendiz, es decir, la escritura, será la más eficaz. Se estudian dos variables principales: el tipo de tarea y el número necesario de exposiciones a la palabra para que esta sea incorporada; y las variables sociales: sexo de los informantes, dominio de otras lenguas, nivel sociocultural e interés y motivación por aprender la lengua meta. Como muestra de la investigación global, exponemos en este artículo los experimentos 
realizados en Madrid, con informantes de L1 inglés, en situación de español como lengua comunitaria.

Palabras Clave: Aprendizaje de español como lengua no materna o lengua meta, aprendizaje de vocabulario, niveles de procesamiento, tipo de tarea, número de exposiciones

\section{ABSTRACT}

This paper presents an experimental research focused on learning vocabulary in Spanish as target language, which is being developed at various locations and different learning contexts: Spain, Brazil and USA. The objective is to determine the best way to present a new word to non-native speakers in order to be more easily incorporated into their mental lexicon. We use three types of activities, which are common in teaching second languages: selection of definitions, selection of examples, and writing sentences. The first hypothesis is that the task that requires more processing effort and involvement load of the learner, i.e. writing, will be the most effective one. We study two main variables: the type of task and the required number of exposures to the word to be learnt, and social variables: sex of the subjects, knowledge of other L2, sociocultural level, and interest and motivation to learn the language. As an example of the global research, in this article we present the experiments carried out in Madrid, with informants whose L1 is English, and who are learning Spanish at L2 context.

KEY WORDS: Learning Spanish as target language, learning vocabulary, levels of processing, type of activities, number of exposures

\section{LA INVESTIGACIÓN GLOBAL}

Presentamos en estas líneas el proyecto de una investigación experimental, enmarcada en el ámbito de la Lingüística aplicada a la enseñanza de lenguas concretamente centrada en el aprendizaje del léxico en español como lengua no materna o lengua meta $(\mathrm{E} / \text { Lmeta })^{1}$.

Esta investigación es múltiple en sus fuentes de obtención de datos, ya que cuenta con varios puntos de actuación que actualmente sirven de base para la elaboración de trabajos de investigación y tesis doctorales en el Departamento de Lengua Española y Lingüística General de la Universidad Nacional de Educación a Distancia, de España.

Siempre nos ha parecido de notable utilidad para la enseñanza de lenguas meta o no maternas investigar cómo debía presentarse un término o vocablo a un hablante no nativo para que fuera más sensible y permeable a su aprendizaje. En estos momentos se están haciendo las siguientes aplicaciones de este modelo, que se encuentran en la recogida de datos:

1. Investigación con grupos de lengua materna inglés en un contexto en el que el español es la lengua de comunicación (España),

${ }^{1}$ Aplicamos el término lengua meta en el sentido de una lengua objeto de aprendizaje, ya sea en entornos heterosiglóticos (lengua extranjera o LE) u homosiglóticos (lengua segunda o L2). Para una explicación más amplia de su significado remitimos al Diccionario de términos clave de ELE (http://cvc.cervantes.es/ensenanza/ biblioteca_ele/diccio_ele/diccionario/lenguameta.htm). 
2. Investigación con grupos de lengua materna portugués en un contexto en el que el español no es la lengua de comunicación (Brasil), y

3. Investigación con grupos de lengua materna inglés en un contexto en el que el español no es la lengua de comunicación y, si es posible, con hablantes de español lengua heredada (EE. UU. $\left.{ }^{2}\right)$.

A estos tres tipos de universo de aplicación del modelo de análisis, se añadirá un grupo en contexto de español como lengua profesional (fines específicos).

\section{Presupuestos y antecedentes de La inVestigación Global}

En el proyecto general matriz de esta investigación, partimos de unos presupuestos básicos que, desde la coordinación, sientan las bases para unificar el trabajo de todos los investigadores que aplican el experimento. Se asumen definiciones de una compleja red conceptual en este campo, como son adquisición y aprendizaje del léxico, competencia léxica, competencia semántica, lengua (no) comunitaria, lengua meta, lexicón mental, vocabulario..., y que, conjuntamente con la metodología y el procedimiento, permitirán aunar y comparar los resultados de todos los participantes.

Sobre los antecedentes de la aplicación de este modelo de estudio de aprendizaje del léxico, los primeros trabajos fueron realizados por Coomber, Ramstad y Sheets (1986), que llevaron a cabo un experimento con hablantes de lengua materna inglés, en el Concordia College de Minesota, para ver qué actividad de aprendizaje de léxico nuevo en la L1 era la más efectiva. A su vez estos investigadores recogen estudios anteriores en los que se ponía de manifiesto que el número de exposiciones necesarias para que una palabra pasara a formar parte del lexicón mental variaba dependiendo de la habilidad del estudiante, de su motivación y de la dificultad de la palabra ${ }^{3}$.

En ese tercio final del siglo XX, también debemos mencionar como aportación significativa a este campo de estudio la hipótesis de los niveles de procesamiento, de Craik y Lockhart (1972), que enunciaba la relación entre la profundidad de análisis y la retención de las palabras. Según estos autores, la retención de una palabra dependía de con cuánto detalle el alumno elaborara el material de estudio durante la codificación y la práctica; consecuentemente, si el análisis era más detallado o había más elaboración, se retendría más cantidad de material verbal. Aunque esta teoría ha sido modificada y ampliada posteriormente, sus planteamientos básicos se consideran punto de partida de numerosos estudios.

\footnotetext{
${ }^{2}$ Decir que el español no es lengua de comunicación en los EE. UU. es una cuestión muy discutible. No se puede considerar que el español es «ajeno» en un país donde los hispanohablantes superan los 40 millones. No obstante, para esta investigación es importante establecer distinciones entre las comunidades que rodean a los sujetos que realizan el aprendizaje. La localización de los grupos de trabajo en Virginia nos permite adjudicarle esta etiqueta al español. Consideraciones aparte merecerán, en su momento, los sujetos de español lengua heredada.

${ }^{3}$ Coomber, Ramstad y Sheets (1986: 281-285) citan a una serie de autores, cuyas referencias bibliográficas consideran sus predecesores en investigaciones de adquisición de léxico, que también hemos revisado con el fin de conocer el estado de la cuestión en ese momento; y en su mayoría pertenecientes al campo de la psicología cognitiva: Benton, Glover y Plake (1984), Bower y Karlin (1974), Craik y Lockhart (1972), Deighton (1960), DiVesta y Peverly (1984), Emig (1977), Glover, Plake y Zimmer (1982), Hyde y Jenkins (1969), Jacoby y Craik (1979), McDaniel (1984), Peper y Mayer (1978), Ross (1981), Slamecka y Fevreiski (1983), Slamecka y Graf (1978), Tulving (1979), Underwood y Schulz (1960).
} 
Por esa época, Emig (1977) propone la escritura como un medio efectivo de aprendizaje; para la autora representa la forma única de aprendizaje, ya que, como proceso y producto tiene una serie de atributos que corresponden únicamente a ciertas estrategias potentes de aprendizaje: «Writing serves learning uniquely because writing as processand-product possesses a cluster of attributes that correspond uniquely to certain powerful learning strategies» (Emig, 1977: 122) ${ }^{4}$.

Teniendo presentes los planteamientos anteriores, Coomber, Ramstad y Sheets (1986) formularon su hipótesis: la forma más efectiva de incorporar una palabra al lexicón mental será la que exige un mayor nivel de procesamiento. Entonces, emplean en su experimento tres tipos de tareas: dos de ellas exigen ciertos niveles diferentes de procesamiento por parte del aprendiz (escritura de oraciones con palabras estímulo -mayor nivel- y selección de ejemplos en los que se emplean dichas palabras -menor nivel-); y una tercera tarea (selección de la definición correcta) en la que no se precisa un nivel profundo de procesamiento:

The present study investigated the effects of composing and of rehearsal with examples on retention of word meaning. While these two types of elaboration are obviously not the only ones available, they have held clear theoretical interest. These two approaches were compared to a group of students studying definitions-a traditional approach to vocabulary teaching that does not call for deeper-level processing (Coomber, Ramstad y Sheets, 1986: 285).

A partir del trabajo de Coomber, Ramstad y Sheets (1986), otras investigaciones han ido aplicando ese u otro modelo semejante hasta la actualidad con muestras de informantes no siempre suficientes. Se pueden citar como relevantes para el interés de nuestro proyecto los siguientes estudios, pues en todos ellos se incluye, al menos, una tarea que integra la escritura como forma de aprendizaje de nuevas palabras, que es el ejercicio que, según la hipótesis de los investigadores de Minesota, será más efectivo para incorporar nuevos vocablos al lexicón mental:

- En español/lengua materna: Puerto Rico - Matanzo Vicens (1991; 1999); España - Reyes Díaz (1995).

- En inglés/lengua meta: Israel y Holanda - Hulstijn y Laufer (2001); Laufer (2001; 2003); Japón - Webb (2002; 2005); Japón - Browne (2003); EE. UU. - Folse (2006); Canadá - Pichette, De Serres y Lafontaine (2012).

- En español/lengua meta: España - Benítez Pérez, Andión Herrero y Fernández López (1996); EE. UU. - Barcroft (2004; 2006; 2007); España - San Mateo Valdehíta (2005); EE. UU. - Keating (2008); Alemania - Agustín Llach (2009).

\section{MÉTOdOS DE APRENDIZAJE E HIPÓTESIS}

El experimento en el que se centra este proyecto de investigación consiste en realizar una serie de test en los que se comprueba si los estudiantes aprenden 10 palabras estímulo.

\footnotetext{
${ }^{4}$ Aunque Peper y Mayer (1978) corroboraron la idea de Emig con un experimento realizado con estudiantes que toman apuntes en clase y con otros que no lo hacen, esta hipótesis ha sido objeto de muchas refutaciones y críticas. En lo que sí parecen estar de acuerdo los investigadores es en que hay ciertas estrategias que caracterizan el aprendizaje realizado con éxito, y entre ellas están el refuerzo y la retroalimentación o feedback.
} 
Estas palabras son en realidad pseudopalabras o palabras inventadas, de igual longitud silábica, con características acentuales y gramaticales controladas; sus significados también son inventados y acordes con los especiales destinatarios de los experimentos (aprendices de español como lengua meta). La prueba consiste en la utilización de un determinado tipo de actividad o método de aprendizaje en tres subgrupos diferentes de aprendices (cada subgrupo practica con un tipo de ejercicio): método de selección de definiciones, método de selección de ejemplos y método de redacción de oraciones (véase una muestra de las actividades más adelante, en el apartado 6.2), que son los mismos que emplearon para estudios sobre aprendizaje de vocabulario de lengua materna Coomber, Ramstad y Sheets (1986) (inglés), y Matanzo Vicens (1991; 1999) y Reyes Díaz (1995) (español).

La hipótesis principal que intentamos probar o refutar es si la actividad que más facilita el aprendizaje de vocabulario es también la que le exige más grado de esfuerzo y procesamiento al aprendiz. En este caso, se trata de la escritura de oraciones originales, tal y como ocurre cuando aprendemos nuevas palabras en la lengua materna.

\section{VARiables de LA INVESTigación}

En la investigación consideramos dos variables principales: método empleado en el aprendizaje y número de exposiciones necesarias para aprender la nueva palabra. Estas variables serán cotejadas en relación con otras variables válidas para todos los puntos donde la investigación se está llevando a cabo: categoría gramatical de las palabras, sexo de los informantes, dominio de otras lenguas, nivel sociocultural e interés y motivación por aprender la lengua meta.

La realidad social-educativa de cada país y el lugar en el que se realiza la investigación hacen que existan otras variables de aplicación opcional, que pueden quedar neutralizadas según el caso. Hablamos de: la edad (aunque resulta interesante por sus implicaciones en la madurez cognitiva del individuo, puede no ser pertinente en grupos de estudio con edades muy uniformes); la lengua materna (solo entra en las coordenadas de relación cuando se trata de grupos multilingües y en la comparación global de los resultados, pues los grupos serán de lenguas maternas diversas); el nivel de español (se preestablece que sea superior al A1 -nivel inicial, principiante, descrito en el Marco común europeo de referencia para las lenguas: aprendizaje, enseñanza y evaluacióny será válido si los grupos de estudio no tienen un nivel uniforme); y la procedencia escolar o tipo de escuela (cobra significado cuando se pueden estudiar a la vez grupos formados en centros privados y públicos). Así, cada experimento particular considera un número de las variables básicas, y puede incrementarlas atendiendo a los condicionamientos concretos de cada aplicación.

En la investigación global añadimos la variable del contexto de aprendizaje (español lengua comunitaria / español lengua no comunitaria), que no puede ser atendida hasta que no estén los resultados de las diferentes localizaciones de los estudios, y en cuya valoración participarán todos los investigadores vinculados.

En el análisis de las variables de la investigación, la corrección de los test realizados por los informantes es manual o con plantillas en el caso de las actividades de selección de definiciones y de ejemplos. Los resultados de los test de cada sujeto, 
los de cada subgrupo y los generales, cuando dispongamos de los datos de todos los experimentos, se organizan en hojas de cálculo que permiten operaciones parciales, medias aritméticas y medianas a lo largo del proceso de recogida de datos, para así poder ver la evolución de cada informante y del experimento en general (incluyendo los datos de las variables sociales). Los datos se exportan al programa de estadística SPSS, para realizar el análisis de varianza (ANOVA) y la prueba T-Student, según sea el número de variantes que establece cada variable (por ejemplo, en el caso del análisis del número de aciertos en relación con el sexo masculino o femenino de los sujetos, se emplea la T-Student).

\section{OBJETIVOS Y APLICACIÓN PRÁCTICA}

Los objetivos de la investigación general o global están orientados a:

1. Determinar el método más eficaz para aprender nuevas palabras, relacionado con la velocidad de incorporación de las mismas al lexicón mental;

2. Establecer el número mínimo de exposiciones a un término o vocablo para que se produzca la incorporación del mismo al lexicón mental; y

3. Conocer qué tipo de tarea, medida por su grado de complejidad, puede realizar el aprendiz con las palabras aprendidas, teniendo en cuenta la diversidad de las tareas y la calidad de la producción exigida.

Este último objetivo es especialmente interesante, pues diversifica la aplicación del procedimiento en algunos de los experimentos proyectados.

La aplicación práctica de las conclusiones de este proyecto de investigación sobre la eficacia de métodos de incorporación léxica en contextos formales de aprendizaje del E/Lmeta estará encaminada a: proponer un diseño y tipología de actividades para el aula y en manuales de español (lengua meta), y planificar y sistematizar el número de veces que un aprendiz de español necesita estar expuesto o trabajar con una palabra para incorporarla a su lexicón mental. Ambas acciones tienen inmediata aplicabilidad en la creación de manuales y materiales de E/Lmeta.

\section{EXPERIMENTO PILOTO}

Como muestra de la investigación general o global, exponemos a continuación el experimento llevado a cabo en Madrid, con sujetos cuya L1 es el inglés, en situación de español como lengua comunitaria. Este experimento ha perseguido como objetivo fundamental probar la pertinencia de la metodología aplicada, prever soluciones razonadas a la casuística de problemas que se den en el proceso de aplicación del experimento y observar el comportamiento de las variables, sobre todo las sociales, en cuanto a la corrección de sus parámetros de medición o escalas y su oportunidad al poder quedar neutralizadas en relación con el comportamiento de las variables principales (método de aprendizaje y número de exposiciones). 


\subsection{Hipótesis, variables de estudio e informantes}

La investigación piloto, llevada a cabo entre 2003-2005, parte de dos hipótesis de trabajo:

1. Existe relación entre el método empleado para aprender léxico y la incorporación del mismo al lexicón mental.

2. En una escala de mayor a menor rango de eficacia, los métodos se ordenan de la siguiente manera: redacción de oraciones, selección de definiciones y, por último, selección de ejemplos.

El ordenamiento de los métodos presentado en la segunda hipótesis está formulado teniendo en cuenta los resultados obtenidos en aplicaciones con referencia a la lengua española, a saber, los de Puerto Rico y Las Palmas de Gran Canaria, por Matanzo Vicens (1991; 1999) y Reyes Díaz (1995), respectivamente. Los datos aportados por estas investigadoras difieren de los de Coomber, Ramstad y Sheets (1986), en los cuales la selección de ejemplos fue más efectiva que la de definiciones.

En definitiva, el objetivo del experimento de Madrid es llegar a la conclusión de cuál es el tipo de actividad más eficaz para incorporar palabras nuevas al lexicón mental y determinar el número de exposiciones requerido; así como comprobar la influencia de las diferentes variables sociales y lingüísticas en el proceso de aprendizaje.

Las variables sociales que se tuvieron en cuenta fueron: el sexo, la procedencia escolar (centros públicos, privados o ambos), el nivel sociocultural de la familia, el número de años que llevaban estudiando español y su interés y motivación por aprenderlo (motivos profesionales, culturales y familiares o personales). Para recopilar esta información de los participantes en el estudio, antes de realizar los test propiamente dichos rellenaron un cuestionario donde debían reflejar estos datos (véase anexo). Por otro lado, las variables lingüísticas fueron la categoría gramatical de las palabras y el tipo de actividad o método de aprendizaje. La variable dependiente fue el número de exposiciones necesarias para incorporar el nuevo vocablo al lexicón mental.

La muestra de informantes quedó constituida por 23 universitarios estadounidenses, de entre 20 y 21 años, que no habían estudiado otras lenguas aparte de español y que tenían un nivel intermedio de esta L2. Aunque la muestra es reducida, bastaba para hacer las comprobaciones metodológicas necesarias, permitir el entrenamiento de los investigadores en el procedimiento de aplicación del experimento y probar la viabilidad del mismo en su aplicación al ámbito del español como lengua segunda o extranjera (el caso de Madrid, como segunda lengua).

\subsection{Metodología y muestra de las actividades}

Las pruebas se aplicaron tal y como hicieron Matanzo Vicens $(1991 ; 1999)$ y Reyes Díaz (1995) con estudiantes de español como lengua materna, siguiendo en parte el estudio de Coomber, Ramstad y Sheets (1986), con alumnos de inglés como lengua materna. Nos detendremos en los pasos seguidos, dados su detalle y complejidad. 
Primero, se reparte un listado de 20 palabras (10 palabras estímulo - barmil, casdel, cienge, leiver, sostro, tarjal, teifor, trelfa, trilen, zulvar- y 10 de baja frecuencia ${ }^{5}$-baltra, fauces, filial, juglar, lacrar, nuncio, plagar, plagio, ristra, viable- ordenadas alfabéticamente) con sus correspondientes definiciones, que funciona a modo de diccionario, y el investigador lo lee en voz alta. El diccionario se recoge y, después, se entregan los ejercicios a los aprendices teniendo en cuenta que había tres tipos diferentes de métodos o actividades de aprendizaje: así, un grupo aprende las palabras trabajando con selección de definiciones, otro con selección de ejemplos en los que se usaba un equivalente de la palabra estímulo y otro escribiendo oraciones con dichas palabras. Para no afectar la motivación de participación en el experimento es importante que los informantes no sepan cuál es el objetivo del experimento ni que las 10 palabras estímulo han sido creadas para este fin (entiéndase, que son inventadas).

A continuación ofrecemos una muestra de las actividades utilizadas en el experimento que ejemplifica de manera concreta en qué consisten los ejercicios a los que deben responder los informantes:

- El grupo que trabaja con el método de definiciones debe seleccionar la definición correcta de la palabra estímulo:

Escribe la palabra TARJAL al lado de la definición que le corresponda:

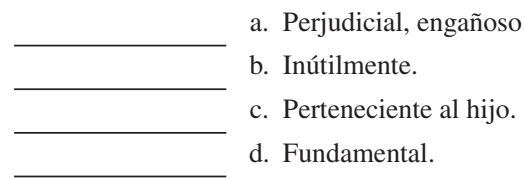

- El grupo que trabaja con el método de ejemplos debe seleccionar el ejemplo en el que se utilice correctamente un equivalente de la palabra estímulo:

Escribe la palabra TARJAL al lado del ejemplo que le corresponda:

a. Unos jóvenes obligaron a Luis a cometer un delito grave.

b. Trató inútilmente de evitar el accidente.

c. Ese joven les falta el respeto a sus padres y a sus profesores.

d. La reforma educativa es un asunto que ha provocado muchas discusiones en España.

- El grupo que trabaja con el método de redacción de oraciones debe contestar a la pregunta con una oración completa en la que se utilice la palabra estímulo:

Contesta a la siguiente pregunta con una oración completa. Utiliza la palabra indicada EN MAYÚSCULAS Y NEGRITA y subráyala. Tu contestación debe demostrar que conoces el significado de dicha palabra:

Cuando trabajas y no consigues los resultados esperados, ¿crees que trabajaste TARJAL? ¿Por qué?

\footnotetext{
${ }^{5}$ Estas palabras fueron escogidas al azar entre las que poseían un número pequeño de ocurrencias en corpus consultados (CORDE y CREA). Además, se hizo una verificación previa sobre su conocimiento con grupos de aprendices de ELE.
} 
El investigador, una vez pasado el tiempo asignado para que los informantes realicen cada ítem, lee la palabra correcta y su definición, de manera que se produce una retroalimentación. Esta acción responde a que la mayor parte de los investigadores está de acuerdo en que el refuerzo y la retroalimentación o feedback están presentes en el aprendizaje realizado con éxito; de ahí que sea parte esencial en este estudio.

El indicador de que el alumno conoce una palabra es contestar correctamente tres veces consecutivas al mismo ítem en las diferentes sesiones en las que se lleva a cabo el experimento; $\mathrm{y}$, hasta lograr este objetivo, se repitieron los test a lo largo de tres meses y medio, con un intervalo de realización de entre tres y cinco días.

\subsection{Resultados obtenidos}

Tras analizar los resultados de los test obtuvimos el promedio del número de exposiciones necesarias para incorporar una palabra según las diferentes variables.

Por un lado, se comprobó que las variables sociales sexo, nivel sociocultural y procedencia escolar pre-universitaria habían quedado neutralizadas desde el punto de vista estadístico (tablas 1-3). En el caso de la variable sexo (tabla 1), la prueba de la T de Student indicó que la probabilidad de error fue superior a 0,05: t $(21)=0,771 \mathrm{p}=0,969$; así que la conclusión es que, con un índice de confianza del 95\%, la diferencia entre los resultados obtenidos por hombres y mujeres no es significativa.

Tabla 1. Resultados obtenidos según la variable sexo

\begin{tabular}{|c|c|c|}
\hline \multirow{2}{*}{ Sexo } & Hombres & Mujeres \\
\cline { 2 - 3 } & 4,580 & 4,562 \\
\hline
\end{tabular}

Lo mismo ocurre con la variable procedencia escolar pre-universitaria (tabla 2): en este caso, aplicamos el análisis de varianza (ANOVA), pues la variable establece tres variantes (el grupo de sujetos que ha asistido a la escuela pública, el de los que proceden de la escuela privada y el de los que se han formado en ambos sistemas), y el resultado es que también la probabilidad es superior a 0,05: $\mathrm{F}(2,20)=2,664 \mathrm{p}=0,094$; con lo cual, con un índice de confianza del $95 \%$, la procedencia escolar tampoco es un factor diferenciador en la incorporación de nuevas palabras al lexicón mental. Sin embargo, si reducimos el margen de confianza a 90\%, la diferencia sí que es significativa.

Tabla 2. Resultados obtenidos según la variable procedencia escolar pre-universitaria

\begin{tabular}{|c|c|c|c|}
\hline $\begin{array}{c}\text { Procedencia escolar } \\
\text { (pre-universitaria) }\end{array}$ & Pública & Privada & Ambas \\
\cline { 2 - 4 } & 4,244 & 4,45 & 5,6 \\
\hline
\end{tabular}

Los resultados de la variable nivel sociocultural son exactamente iguales en ambos grupos (tabla 3). 
Tabla 3. Resultados obtenidos según la variable nivel sociocultural

\begin{tabular}{|c|c|c|}
\hline \multirow{2}{*}{ Nivel sociocultural } & Medio & Medio-alto \\
\cline { 2 - 3 } & 4,6 & 4,6 \\
\hline
\end{tabular}

Puesto que en este experimento se mide la capacidad de un aprendiz para responder a un entrenamiento específico, no sorprende que no influya ningún factor social (López Morales, 1993: 17; San Mateo Valdehíta, 2005: 88) y las expectativas se orienten hacia la eficacia de las actividades de aprendizaje en sí mismas.

Si analizamos los resultados teniendo en cuenta el número de años que los alumnos llevan estudiando la L2 (tabla 4), vemos que los que más exposiciones necesitan son los que llevan de siete a ocho años. Ello nos puede llevar a pensar que el aprendizaje se ha estancado y les cuesta más ampliar el vocabulario. El análisis de varianza nos confirma que la diferencia entre los resultados no es significativa con un nivel de confianza del 95\%, ya que la probabilidad de error es superior a 0,05 : $F(2,20)=0,772$ $\mathrm{p}=0,476$.

Tabla 4. Resultados obtenidos según la variable número de años de estudios de español

\begin{tabular}{|c|c|c|c|}
\hline \multirow{2}{*}{$\begin{array}{l}\text { Número de años de } \\
\text { estudios de español }\end{array}$} & De 4 a 6 & De 7 a 8 & Más de 8 \\
\hline & 4,522 & 4,957 & 4,243 \\
\hline
\end{tabular}

En los resultados de la variable interés y motivación por aprender la lengua (tabla 5), llama la atención el aumento en el número de exposiciones necesarias cuando se trata de informantes con más motivos para aprender la lengua: es posible que el exceso de expectativas despierte demasiado interés y obstaculice el aprendizaje siendo estresante para el alumno. Tampoco hay que olvidar que el número de sujetos en este trabajo no es muy amplio y que solo hay una persona que señala motivos para aprender español en las tres categorías que hemos utilizado para clasificar las respuestas: motivos profesionales, familiares y culturales. Este valor (7,7 exposiciones), que bien podría calificarse de atípico (outlier), desvirtúa el análisis estadístico, ya que entre el resto de los grupos las diferencias son mínimas y es un único informante el que aporta el dato discordante.

Tabla 5. Resultados obtenidos según la variable interés y motivación por aprender la L2

\begin{tabular}{|l|c|}
\hline \multicolumn{2}{|l|}{ Interés y motivación por aprender la lengua } \\
\hline Profesional & 4,267 \\
\hline Cultural & 4,8 \\
\hline Profesional y cultural & 4,25 \\
\hline Profesional y personal & 4,05 \\
\hline Personal y cultural & 4,7 \\
\hline Profesional, personal y cultural & 7,7 \\
\hline
\end{tabular}


En cuanto a la categoría gramatical (tabla 6), es el adverbio, es decir, la categoría invariable, la que los alumnos incorporaron más rápidamente, mientras que la que posee más accidentes morfológicos, el verbo, fue la que requirió un mayor número de exposiciones. Mediante la prueba $\mathrm{T}$ de Student comparamos por pares los resultados de las categorías y se confirma que la diferencia entre ellos no es significativa con un nivel de confianza del $95 \%$, ya que la probabilidad de error es superior a 0,05 en todos los casos: entre nombre y verbo, $\mathrm{t}(22)=-1,455 \mathrm{p}=0,160$; entre nombre y adjetivo, $\mathrm{t}(22)=-1,262$ $\mathrm{p}=0,220$; entre nombre y adverbio, $\mathrm{t}(22)=-0,401 \mathrm{p}=0,692$; entre verbo y adjetivo, $\mathrm{t}$ $(22)=0,589 \mathrm{p}=0,526$; entre verbo y adverbio, $\mathrm{t}(22)=-1,726 \mathrm{p}=0,098 \mathrm{y}$ entre adjetivo $\mathrm{y}$ adverbio, $\mathrm{t}(22)=-1,251 \mathrm{p}=0,224$.

Tabla 6. Resultados obtenidos según la variable categoría gramatical de las palabras estímulo

\begin{tabular}{|c|c|}
\hline $\begin{array}{c}\text { Categoría gramatical de } \\
\text { las palabras }\end{array}$ \\
\hline Adverbio & \multicolumn{1}{c|}{4,3} \\
\hline Nombre & 4,4348 \\
\hline Adjetivo & 4,6667 \\
\hline Verbo & 4,8261 \\
\hline
\end{tabular}

Por último, según la variable método (tabla 7), el más eficaz fue el de selección de definiciones, después el de redacción de oraciones y, por último, el de selección de ejemplos. El análisis de varianza indica que la probabilidad es superior a 0,05: F $(2,20)$ $=2,640 \mathrm{p}=0,096$; por lo que, con un índice de confianza del 95\%, la diferencia entre los resultados obtenidos con cada una de las actividades no es significativa; pero sí lo sería si reducimos el margen de confianza al $90 \%$.

Tabla 7. Resultados obtenidos según la variable método de aprendizaje

\begin{tabular}{|c|c|c|c|}
\hline \multirow{2}{*}{ Método } & Definiciones & Oraciones & Ejemplos \\
\cline { 2 - 4 } & 3,91 & 4,90 & 4,94 \\
\hline
\end{tabular}

\subsection{Comparación de los resultados con los de investigaciones previas}

Los resultados obtenidos en el estudio piloto no coinciden exactamente con los de los experimentos anteriores en los que se trabajaron con estos tres mismos tipos de actividades: en el estudio de Coomber, Ramstad y Sheets (1986), el método más eficaz fue el de redacción de oraciones; luego, el de selección de ejemplos y, por último, el de selección de definiciones. Se cumplía, por tanto, su hipótesis de trabajo según la cual la actividad de escritura es la más productiva, ya que es la que requiere un mayor nivel de procesamiento; y la selección de definiciones, al ser la que menos procesamiento conlleva, es la menos eficaz:

The investigators hypothesized that the more deeply the words and their definitions were processed, the more successfully students would retrieve those definitions on a memory 
test. Definitions were deemed to require the least processing while examples would require somewhat more processing. Sentence-composing, clearly a generative activity, would require the most processing [...] (Coomber, Ramstad y Sheets, 1986: 290).

En los trabajos de Matanzo Vicens (1991; 1999) y Reyes Díaz (1995), también fue la redacción de oraciones la actividad más eficaz, seguida de la selección de definiciones y de la selección de ejemplos; por lo tanto, cambia el orden de la segunda y la tercera, respecto a los resultados del primer estudio de Coomber, Ramstad y Sheets (1986).

En nuestro experimento piloto, hemos corroborado la hipótesis primera, sobre la existencia de una relación entre el método empleado para aprender léxico y la incorporación del mismo, si bien, la diferencia entre los resultados de las tres actividades no es grande. Pero no se ratifica la segunda hipótesis, pues la selección de definiciones fue la forma más eficaz de incorporar nuevas palabras.

Para estudiantes de una L2, la ventaja de la actividad de selección de definiciones sobre la de seleccionar ejemplos y la de escribir oraciones se podría explicar por varias razones: en primer lugar, porque el trabajo con definiciones y el uso del diccionario es una práctica habitual en el aprendizaje de vocabulario en lenguas segundas o extranjeras, por lo que no resulta extraño que los alumnos estén habituados a este tipo de actividad.

Por otro lado, dado el reducido número de informantes, hay que tener presente que aquí también tenemos un valor atípico, pues:

[...] solamente en el grupo que ha trabajado con definiciones hay una persona que ha sido capaz de contestar correctamente las diez preguntas desde el principio. [...] Damos por hecho que en todo tipo de encuestas siempre hay informantes «brillantes»; en nuestro caso, donde el número de informantes es tan reducido, realmente esta capacidad excepcional de una sola persona puede desvirtuar los resultados y hacer que un método de aprendizaje resulte mucho más económico que otro (San Mateo Valdehíta, 2005: 86).

Finalmente, cabe señalar que la diferencia entre los promedios de exposiciones obtenidos con los tres métodos no es significativa desde el punto de vista estadístico, con un nivel de confianza del $95 \%$, como hemos indicado antes.

La media del número de exposiciones que necesitaron los informantes para incorporar una palabra nueva fue de 4,6. Esta cifra se acerca a la obtenida por Reyes Díaz $(1995)(4,8)$ y difiere bastante de la de Matanzo Vicens (1991) (10,4). Reflexionamos sobre que probablemente la edad de los informantes es el factor diferenciador respecto de Matanzo Vicens (1991); además, claro está, de que en este caso la investigación se hizo en L1. No podemos ofrecer comparativas con el estudio de Coomber, Ramstad y Sheets (1986) pues estos investigadores no utilizaron la variable número de exposiciones necesarias para aprender una nueva palabra.

\subsection{Conclusiones del estudio piloto}

Las conclusiones del estudio piloto, más allá de las que se desprenden de los resultados mismos del experimento, fueron -como esperábamos- fundamentalmente de naturaleza metodológica:

1. Las variables sociales empleadas eran las adecuadas y se mantendrían en la investigación posterior - pues, aunque algunas quedaron neutralizadas, consideramos 
conveniente seguir comprobando si ejercen algún tipo de influencia sobre el aprendizaje de vocabulario-.

2. La puesta en práctica del experimento -que es una réplica del de Matanzo Vicens (1991); reproducido después también por Reyes Díaz (1995)- con sesiones consecutivas podía influir negativamente en el proceso de aprendizaje. Al tratar de determinar el número de exposiciones necesarias para que una palabra pasara al lexicón mental y tener que repetir un mismo test un número elevado de veces (tantas como fueran necesarias hasta que utilizaran correctamente cada palabra tres veces consecutivas, como hemos dicho antes), los informantes se muestran agotados provocando en ellos desmotivación.

Haber detectado esta incidencia de implementación de los test nos ha permitido retomar en otras aplicaciones del proyecto la metodología usada por Coomber, Ramstad y Sheets (1986). Es decir, con el objetivo de saber cuál es el tipo de actividad más efectivo y con el que se aprende un número mayor de palabras, suprimimos la variable número de exposiciones necesarias para, de esta forma, poder valorar la efectividad de la variable interés y motivación, sin que la mecánica del experimento tenga efectos negativos en el aprendizaje, e incorporamos la variable actividad de entrenamiento, como hicieron los investigadores de Minesota.

La relevancia del estudio piloto está, por tanto, en comprobar la eficacia de una metodología con antecedentes tan dispares, y de ahí su importancia.

\section{AmpliaCión DEL ESTUdio PILOTO}

\subsection{Informantes y procedimiento}

Así pues, hemos puesto en práctica una segunda investigación aún sin terminar, con 150 universitarios estadounidenses (L1 inglés), de 19 a 21 años, de nivel intermedio de español y en situación de inmersión en la lengua meta en Madrid, donde participan en un programa universitario en el que se les exige, al menos, un nivel B1 de español. Ello garantiza la comprensión fiable de todo el material empleado en el experimento (diccionario, definiciones del listado y ejercicios).

El material de trabajo es el mismo que empleamos en el experimento anterior: el cuestionario previo para obtener la información personal sobre cada informante, el listado con las palabras estímulo y de baja frecuencia con sus definiciones correspondientes -es decir, el diccionario completo- y los test (actividades). La diferencia fundamental con respecto al estudio piloto es que en este caso se incluyen ejercicios previos de entrenamiento que consisten en dividir a los informantes en tres grupos, y que cada uno trabaje con un tipo de actividad de entrenamiento: uno con selección de definiciones, otro con selección de ejemplos y el tercero debe escribir oraciones en las que emplee las palabras estímulo. Se realizan dos series seguidas de actividades de entrenamiento: en la primera, los estudiantes pueden consultar el diccionario de palabras si lo necesitan; mientras que en la segunda serie realizan los ejercicios sin disponer del diccionario. Esto supone un mayor grado de dificultad y apoyo memorístico.

Por lo demás, se procede igual que en el estudio piloto, el investigador cronometra el tiempo, y va dando retroalimentación (lee la palabra y la definición correcta) una vez que pasa el tiempo asignado a cada ítem. 
Tras los entrenamientos, los sujetos realizan un postest. Esta prueba consiste en una serie de ejercicios que se lleva a cabo sin poder consultar el diccionario, y sus resultados son los que realmente se puntúan. La peculiaridad del postest es que todos los grupos hacen el mismo, que incluye actividades de los tres tipos de modelos: selección de definiciones y de ejemplos, y redacción de oraciones, aunque los sujetos se hayan entrenado solo con un tipo de ellos, como se ha explicado líneas más arriba. Ello nos permitirá observar cuántos aciertos tienen los sujetos en cada tipo de ejercicios y qué relación tiene su grado de éxito con el entrenamiento que han recibido.

\subsection{Corrección de los postest}

Al contabilizar los resultados de los ejercicios de las secciones del postest en las que trabaja con la selección de ejemplos y la selección de definiciones no se han planteado dudas para determinar los aciertos y los errores, pues se trata de una prueba objetiva: de las opciones presentadas solo una es correcta. Sin embargo, ha sido preciso establecer una serie de criterios para realizar la puntuación y corrección de las oraciones:

1. Al igual que en la investigación piloto, y en otros experimentos en los que se trabaja con la creación de enunciados, no se tendrán en cuenta los errores ortográficos ni sintácticos. Ejemplo: No sentía trelfa porque he estado en Madrid cinco veces antes y conocía a la ciudad muy bien (informante 2_O).

2. Se considera correcta la oración cuando se utiliza un sinónimo de la palabra estímulo, siempre que esté claro que se comprende el significado. Ejemplo: Sí, yo he asistido varios acontecimientos deportivos en que había muchísima gente (informante 3_E) [muchísima gente por cienge].

\subsection{Consideraciones de interés}

Esta investigación permitirá:

- Establecer el número de palabras aprendidas con cada uno de los tres métodos de instrucción,

- Comprobar la efectividad de cada uno de los métodos: el uso de la palabra aprendida en diferentes contextos y tipos de actividades,

- Analizar la influencia de las variables sociales en el aprendizaje de vocabulario,

- Examinar los resultados a la luz de las diferentes teorías de procesamiento de la información (nivel de procesamiento y profundidad de análisis; hipótesis de la participación o del compromiso) y de incorporación de vocabulario al lexicón mental, y

- Comparar los resultados con los del resto de las investigaciones del proyecto global en el que se inserta.

\section{Conclusiones}

Los estudios experimentales sobre la incorporación de vocabulario al lexicón mental de un estudiante de segundas lenguas tienen importantes aplicaciones didácticas, por eso creemos interesante un estudio global y múltiple, que se elabore desde varios puntos y en diferentes contextos de aprendizaje. 
Una de las investigaciones es la llevada a cabo en Madrid, con universitarios de L1 inglés, en un contexto en el que el español es la lengua de comunicación. Hemos presentado en este artículo un estudio piloto de este trabajo, como muestra de la investigación general, para que sean entendidos más cabalmente la esencia y el procedimiento del experimento.

El estudio piloto, iniciado antes de emprender el experimento con un grupo numeroso de informantes, nos ha permitido probar la metodología y el procedimiento de ejecución y nos ha servido para situar un punto de partida sólido ante antecedentes tan dispares (los resultados de los trabajos realizados en L1 no son homogéneos, y la diferencia entre el número de exposiciones es muy grande); y de ahí su relevancia.

En cuanto al procedimiento de ejecución, gracias a la observación realizada durante el estudio piloto hemos decidido realizar una modificación importante, volviendo al planteamiento del trabajo inicial de Coomber, Ramstad y Sheets (1986): una primera fase de entrenamientos y un postest. Este será el procedimiento que aplicaremos en algunos puntos del experimento general.

Los resultados del estudio piloto indican que el método de definiciones, pese a su escaso «gasto cognitivo», parece estar privilegiado en la aprehensión léxica, al menos en el caso del aprendiz de segundas lenguas; si bien, cabría esperar que la escritura de oraciones, puesto que exige un mayor nivel de procesamiento y de análisis, fuera la manera de aprendizaje más eficaz. Establecer el orden de eficacia entre los métodos resulta uno de los intereses clave de este proyecto.

En cualquier caso, estas conclusiones no serán definitivas hasta que todas las aportaciones a la investigación global tengan disponibles sus resultados. Tenemos hacia ellas unas entusiastas expectativas y esperamos que aporten una idea más o menos clara de qué es realmente útil y eficaz para un estudiante de segundas lenguas a la hora de aprender nuevas palabras.

\section{REFERENCIAS BIBLIOGRÁFICAS}

Agustín Llach, M. ${ }^{a}$ Pilar (2009): «The effect of reading only, reading and comprehension, and sentence writing in lexical learning in a foreign language: some preliminary results», Revista Española de Lingüística Aplicada, 22, págs. 9-33.

BARCROFT, Joe (2004): «Effects of sentence writing in second language lexical acquisition», Second Language Research, 20.4, págs. 303-334.

- (2006): «Can writing a new word detract from learning it? More negative effects of forced output during vocabulary learning», Second Language Research, 22.4, págs. 487-497.

- (2007): «Effects of word and fragment writing during L2 vocabulary learning», Foreign Language Annals, 40.4, págs. 713-726.

Benítez Pérez, Pedro, M. ${ }^{a}$ Antonieta Andión Herrero y M. ${ }^{a}$ Carmen Fernández López (1996): «El aprendizaje del vocabulario en español como lengua extranjera. Proyecto de investigación». En Segoviano, Carlos (ed.): La enseñanza del léxico español como lengua extranjera, Frankfurt-Madrid, Vervuert-Iberoamericana, págs. 140-149.

Benton, Stephen L., John A. Glover y Barbara S. Plake (1984): «Employing adjunct aids to facilitate elaboration in writing», Research in the teaching of English, 18.2, págs. 189-200.

Bower, Gordon H. y Martin B. Karlin (1974): «Depth of processing pictures of faces and recognition memory», Journal of Experimental Psychology, 103.4, págs. 751-757.

BRowne, Charles (2003): Vocabulary acquisition through reading, writing and tasks: a comparison (tesis doctoral inédita), Temple University Japan: http://web.me.com/charlesbrowne1/ 
Eigo_Kyoiku/About_Me_files/3\%29\%20Vocabulary\%20Acquisition\%20Tasks.pdf (20-042012).

CONSEJO DE Europa (2002): Marco común europeo de referencia para las lenguas: aprendizaje, enseñanza y evaluación, Estrasburgo: http://www.cvc.cervantes.es/obref/marco/indice.htm (20-04-2012).

Coomber, James E., David A. Ramstad y Dee A. R. Sheets (1986): «Elaboration in vocabulary learning: A comparison of three rehearsal methods», Research in the teaching of English, 20.3, págs. 281-293.

CrAIK, Fergus I. M. y Robert S. Lockhart (1972): «Levels of processing: A framework for memory research», Journal of verbal learning and verbal behaviour, 11.6, págs. 671-684.

Deighton, Lee C. (1960): «Developing vocabulary: Another look at the problem», The English Journal, 49.2, págs. 82-88.

DiVesta, Francis J. y Stephen T. Peverly (1984): «The effects of encoding variability, processing activity, and rule-examples sequence on the transfer of conceptual rules», Journal of Educational Psychology, 76.1, págs. 108-119.

Emig, Janet (1977): «Writing as a mode of learning», College Composition and Communication, 28.2, págs. 122-128.

FoLSE, Keith S. (2006): «The effect of type of written exercise on L2 vocabulary retention», TESOL Quarterly, 40.2, págs. 273-293.

Glover, John A., Barbara S. Plake y John W. Zimmer (1982): «Distinctiveness of encoding and memory for learning tasks», Journal of Educational Psychology, 74.2, págs. 189-198.

Hulstijn, JAN H. y Batia Laufer (2001): «Some empirical evidence for the Involvement Load Hypothesis in vocabulary acquisition», Language Learning, 51, págs. 539-558.

Hyde, Thomas S. y James J. Jenkins (1969): «Differential effects of incidental tasks on the organization of recall of a list of highly associated words», Journal of Experimental Psychology, 82.3 , págs. $472-481$.

InSTITUto CeRvantes (1997-2011): Diccionario de términos clave de ELE: http://cvc.cervantes. es/obref/diccio_ele/indice.htm.

JACOBY, Larry L. y Fergus I. M. Craik (1979): «Effects of elaboration of processing at encoding and retrieval: Trace distinctiveness and recovery of initial context». En Cermak, Laird S. y Fergus I. M. Craik (eds.): Levels of processing in human memory, Hillsdale, Lawrence Erlbaum Associates, págs.1-21.

Keating, Gregory D. (2008): «Task effectiveness and word learning in a second language: the Involvement Load Hypothesis on trial», Language Teaching Research, 12.3, págs. 365-386.

LAUFER, Batia (2001): «Reading, word-focussed activities and incidental vocabulary acquisition in a second language», Prospect, 16.3, págs. 44-54.

- (2003): «Vocabulary acquisition in a second language: Do learners really acquire most vocabulary by reading? Some empirical evidence», The Canadian Modern Language Review, 59.4, págs. 567-587.

López MoRales, Humberto (1993): «En torno al aprendizaje del léxico. Bases psicolingüísticas de la planificación curricular». En Actas del III Congreso Nacional de ASELE. El español como lengua extranjera: De la teoría al aula, Málaga, ASELE, págs. 9-22.

Matanzo Vicens, Gloria (1991): Vocabulario y enseñanza: Estudio de la relación existente entre los métodos empleados y la incorporación del léxico nuevo a la competencia lingüística de estudiantes universitarios puertorriqueños (tesis doctoral inédita), Universidad de Puerto Rico.

- (1999): «Incorporación léxica y metodología de enseñanza». En Samper Padilla, José Antonio y Magnolia Troya Déniz (coords.): Actas del XI Congreso Internacional de la Asociación 
de Lingüística y Filología de la América Latina. Las Palmas de Gran Canaria, del 22 al 27 de julio de 1996, Las Palmas de Gran Canaria, Universidad de Las Palmas de Gran Canaria, págs. 1623-1628.

McDAnIEL, Mark A. (1984): «The role of elaborative and schema processes in story memory», Memory and Cognition, 12.1, págs. 46-51.

PePER, Richard J. y Richard E. MAYER (1978): «Note-taking as a generative activity», Journal of Educational Psychology, 70.4, págs. 514-522.

PichetTe, Francois, Linda de Serres y Marc Lafontaine (2012): «Sentence reading and writing for second language vocabulary acquisition», Applied Linguistics, 33.1, págs. 66-82.

Reyes Díaz, M.a Josefa (1995): Enriquecimiento de la competencia léxica: análisis estadístico (tesis doctoral inédita), Universidad de Las Palmas de Gran Canaria: http://hdl.handle. net/10553/1999 (20-04-2012).

Ross, Brian H. (1981): «The more, the better? Number of decisions as a determinant of memorability», Memory and Cognition, 9.1, págs. 23-33.

San Mateo Valdehíta, Alicia (2005): Aprendizaje de léxico en español como segunda lengua. Investigación sobre tres métodos (trabajo de investigación Diploma de Estudios Avanzados UNED 2004), Biblioteca redELE: http://www.educacion.gob.es/redele/Biblioteca-Virtual/2005/ memoriaMaster/1-Semestre/SAN-MATEO-UNED.html (20-04-2012).

SLAmeCKA, Norman J. y Jacobo Fevreiski (1983): «The generation effect when generation fails», Journal of verbal learning and verbal behaviour, 22.2, págs. 153-163.

Slamecka, Norman J. y Peter Graf (1978): «The generation effect: Delineation of a phenomenon», Journal of Experimental Psychology: Human learning and memory, 4.6, págs. 592-604.

Tulving, Endel (1979): «Relation between encoding specificity and levels of processing». En Cermak, Laird S. y Fergus I. M. Craik (eds.): Levels of processing in human memory, Hillsdale, Lawrence Erlbaum Associates, págs. 405-428.

Underwood, Benton J. y Rudolph W. Schulz (1960): Meaningfulness and verbal learning, Chicago, Lippincott.

WeBB, Stuart (2002): Investigating the effects of learning tasks on vocabulary knowledge (tesis doctoral inédita), Victoria University of Wellington: http://hdl.handle.net/10063/612 (20-042012).

- (2005): «Receptive and productive vocabulary learning: The effects of reading and writing on word knowledge», Studies in Second Language Acquisition, 27.1, págs. 33-52. 


\section{ANEXO}

\section{CUESTIONARIO REALIZADO A LOS INFORMANTES}

Sexo:

Edad:

¿Cuál es tu lengua materna?

¿Hablas otras lenguas?

¿Cuántos años llevas estudiando español?

¿Dónde lo has estudiado?

¿Por qué estudias español?

¿Cuál consideras que es tu nivel de español? ¿Principiante, nivel intermedio, nivel superior?

Procedencia escolar:

Escuela pública:

Escuela privada:

Ambas:

Información sobre los padres:

Padre:

1. Profesión:

2. Escolaridad:

3. Ingresos anuales:

Madre:

1. Profesión:

2. Escolaridad:

3. Ingresos anuales: 